\title{
Estimation of the Degree of Endolymphatic Hydrops Using Optical Coherence Tomography
}

\author{
Tuukka Karvonen, ${ }^{*}$ \# Yuki Uranishi,,${ }^{* *}$ Tatsunori SaKamoto, ${ }^{* *}$ Yosuke Tona, ${ }^{* *}$ Kazuya Окамото,,${ }^{* * *}$ \\ Hiroshi TAMURA, ${ }^{*}, * *$ Tomohiro KURODA ${ }^{*}, * *$
}

\begin{abstract}
Endolymphatic hydrops is a disorder in which an excessive amount of endolymph fluid causes an increase in the pressure of the endolymphatic system of the inner ear. In cochlea, endolymphatic hydrops can cause stretching in the scala media, a tubular construction that, along with two other similar structures, the scala vestibuli and scala tympani, runs through the cochlea from the base to the top. Visualizing the enlargement of the scala media has traditionally been done by histological study of tissue sections, and the degree can be calculated by comparing the ratio of the area of the scala media to the combined total area of the three compartments. However, this requires the obtainment of tissue samples, and thus is not suited for in vivo measurements, and is a labor intensive process in general. Imaging modalities that have been used to measure endolymphatic hydrops include MRI, $\mu \mathrm{MRI}$ and $\mu \mathrm{CT}$. Recently, optical coherence tomography (OCT) has been applied for visualizing cochlea of mice in vivo. However, just visually discriminating between healthy and afflicted cochlea, and judging the degree of affliction can be difficult. Thus, we aim to establish a new technique for estimating the degree of enlargement of the scala media. We measured two healthy and two diseased cochlea using conventional area calculation, as well as our method, and present results that show that our technique correlates with established standards for measuring the degree of endolymphatic hydrops in cochlea $\left(R=0.977, p=0.5 \times 10^{-7}\right)$.
\end{abstract}

Keywords: optical coherence tomography, endolymphatic hydrops, optical flow.

Adv Biomed Eng. 5: pp. 19-25, 2016.

\section{Introduction}

Ménière's disease is a disease of the inner ear, with symptoms such as hearing loss, vertigo, tinnitus and a sensation of fullness in the ears. Ménière's disease is believed to be caused by endolymphatic hydrops, which refers to the accumulation of excess endolymphatic fluid in the inner ear. Genetic knock out of the Slc26a4 gene is also known to cause endolymphatic hydrops in the inner ear [1].

Traditionally, the diagnosis of Ménière's disease has been through the clinical route, based on observations by a medical professional of the patient's clinical history and symptoms [2]. From histological sections, calculating the degree of endolymphatic hydrops $\left(d_{\text {hyd }}\right)$ can be done by comparing the size of the scala media $\left(A_{\mathrm{med}}\right)$ to the combined sizes of the three vestibules: the aforementioned scala media, the scala vestibuli $\left(A_{\mathrm{ves}}\right)$, and the scala tympani $\left(A_{\mathrm{tym}}\right)$, i.e.

$$
d_{\text {hyd }}=\frac{A_{\text {med }}}{A_{\text {ves }}+A_{\text {med }}+A_{\text {tym }}}
$$

Recently however, methods for directly imaging the cochlea in vivo have emerged. Magnetic resonance imaging (MRI) has been used to image cochlear structure [3]. However, the internal

This study was presented at the Symposium on Biomedical Engineering 2015, Okayama, September, 2015.

Received on August 2, 2015; revised on November 17, 2015; accepted on December 24, 2015.

* Graduate School of Informatics, Kyoto University, Kyoto, Japan.

** Kyoto University Hospital, Kyoto, Japan.

\# 54 Shogoin Kawahara-cho, Sakyo-ku, Kyoto 606-8507, Japan.

E-mail: tuukka@kuhp.kyoto-u.ac.jp structures including the Reissner's and the basilar membranes have been difficult to visualize with MRI because of their small size and thinness. The use of local contrasting agent has started to enable this visualization [4]. Attempts to visualize cochlear structure in vivo using a technique known as optical coherence tomography (OCT) have been made with success $[5,6]$.

OCT is an imaging technique based on near-infrared light that utilizes low-coherence interferometry and the soft-tissue penetrating properties of near-infrared light to measure the reflectivity profile of different points inside tissue samples. Thus it can be used to rapidly obtain micrometer resolution volumetric images of samples. Obtaining these images using OCT, the angle between the Reissner's membrane and the outer wall of the cochlea (the spiral ligament) can be calculated. This study was conducted to find out if the angle between these two planes could be used as a measurement for the existence of endolymphatic hydrops.

To achieve this, we took cochlea samples from mice with and without the Slc26a4 gene, obtained images from them using an OCT machine, and did histology study on the cochlea to obtain a ground truth for the degree of endolymphatic hydrops. After that we compared the results to find out if a correlation exists.

\section{Related works}

Endolymphatic hydrops in Ménière's disease was first discovered independently and simultaneously both by Hallpike \& Cairns [7], and Yamakawa [8]. It has since been imaged by $\mu \mathrm{MRI}$ in [9] as well as MRI in [3, 10, 4]. In [3], the focus was on visualizing fluid progression in the inner ear by injecting gadolinium hydrate diluted with saline in the perilymphatic space. Recently, cochlea have been imaged by OCT devices in $[5,6]$. In the former the authors were interested in imaging the microvascular perfusion in mice using OCT, and in the latter visualizing the effects of endo- 
lymphatic hydrops in cochlea using OCT. The authors in [10] also proposed a scaling method for assessing the degree of endolymphatic hydrops. Grading was based on the relative size of the scala media, with the grades being none $\left(d_{\mathrm{hyd}} \leq 33 \%\right)$, mild $(33 \%<$ $\left.d_{\text {hyd }} \leq 50 \%\right)$ and severe $\left(50 \%<d_{\text {hyd }}\right)$.

Kakigi et al. [11] used isolated and decalcified guinea pig cochlea to obtain full thickness midmodiolar images of cochleae by OCT. With their image, it was possible to apply classical method of calculating the area ratio. However, it is not always possible to obtain such images. Due to the limited imaging depth, normally OCT images include only the lateral area without the modiolus, thus making it difficult to measure the areas of each scala, and to validate if these images are midmodiolar. With our method, only the lateral cochlear areas are required for the calculation and the images do not have to be midmodiolar.

Within the realm of otorhinolaryngology, imaging techniques for diagnosis are increasingly used. MRI and CT scans are common for detecting tumors in cases such as acoustic neuroma [12], dural sinus thrombosis [13] and many others. In ophthalmology, OCT has proven as a superior technique for retinal imaging, such as in detection of macular edema [14] and for detecting macular holes [15], among others. It is also often used for obtaining high resolution cross sectional and volumetric images in eye check ups. Many algorithms have also been developed for automatic retinal layer segmentation, which can help medical professionals in diagnosis, as the amount of data produced by a single volumetric OCT scan can be quite large. A good overview of the different algorithms used for segmentation and their clinical uses can be found in [16].

\section{Theory}

In this section we will go through the structure of the cochlea, as well as an explanation of endolymphatic hydrops. The structure of the cochlea varies depending on the species, and in this study cochlea from mice were used, so in some parts the explanation of the anatomy and the figures will focus on that. After the anatomical background, a short overview of the theory behind OCT is presented, as well as the fundamentals of optical flow.

\subsection{Cochlea}

The cochlea is a spiral structure in the inner ear responsible for converting sound waves into electrical impulses. Together with the vestibular system, which is responsible for the sense of balance, it forms the innermost part of the ear. The cochlea is split into three compartments that run through the length of the spiral structure, the scala vestibuli, scala media, and scala tympani. In humans, the cochlea makes 2.5 turns around it's central axis, the modiolus, while in mice the cochlea is shorter and only makes about 1.5 turns. Sound is transmitted to the cochlear ducts by the stapes, a tiny bone connected to the oval window, which itself is connected to the scala vestibuli. This vibration then travels through to the tip of the cochlea, the helicotrema, where the scala vestibuli and scala tympani are connected, and travels back down the length of the tunnels, vibrating the round window at the end. This vibration, and the connection at the tip of the scala vestibuli and scala tympani causes movement of the two walls separating the three tunnels, the Reissner's membrane and the basilar membrane. This movement peaks at a certain point within the cochlea, depending on the frequency of the sound. This movement is

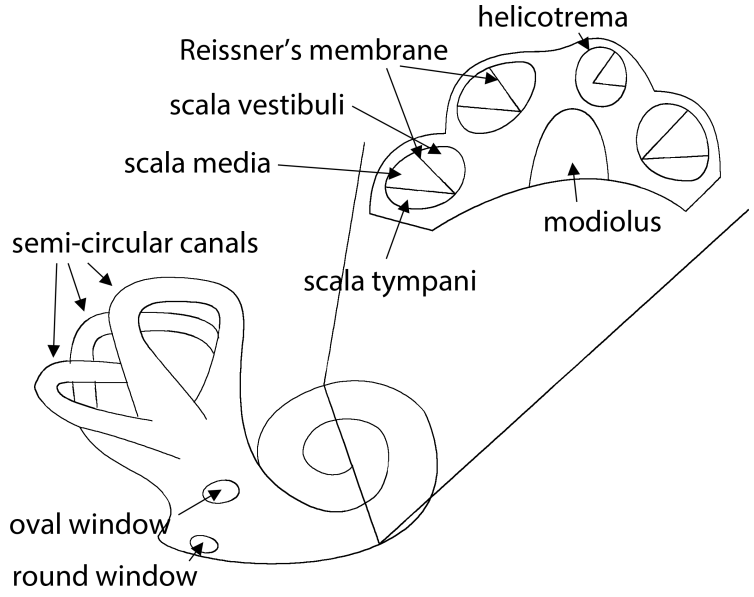

Fig. 1 The structure of the inner ear, including a cross section of the cochlear spiral. Here, the cochlea makes 1.5 turns around the central axis. Important structures are labeled accordingly. Vestibular \& cochlear nerve are missing from the picture.

picked up by the inner hair cells in the organ of Corti, which convert it into electrical impulses that are then eventually sent into the brainstem for processing. [17] The structure of the inner ear and how the cochlea is positioned, as well as structures of the cochlea relevant to this research are shown in Fig. 1.

\subsubsection{Endolymphatic hydrops}

The structure of the cochlea is very delicate, and imbalance in the pressure between the chambers can disturb the function of the ear. This is the case in endolymphatic hydrops, where the pressure in the cochlear duct that houses the endolymph rises, distorting the shape of the walls surrounding it. Symptoms related to endolymphatic hydrops include vertigo, tinnitus, hearing loss and a feeling of congestion in the ear. The symptoms are caused by a buildup of fluid in the inner ear, giving it the name endolymphatic hydrops. This buildup can impair both the aural and vestibular functions provided by the ear. A definite reason for the appearance of endolymphatic hydrops is unknown, but theories range from constrictions in blood vessels, viral infections and genetic variations that cause abnormalities. [18]

No definitive diagnosis exists for endolymphatic hydrops. It is often diagnosed by confirming the existence of specific symptoms and by ruling out other diseases. Treatment includes medication, dietary changes, congnitive therapy and in the worst case, surgery. Prognosis for people suffering from the disease is generally good. [18]

\subsection{OCT}

OCT is an imaging method that utilizes the near-infrared light spectrum and a phenomenon known as interference to capture three-dimensional images from optical scattering media. OCT is analogous to ultrasound, in that the reflection of the light wave is used to measure the distance of the interface from which it reflected. The main difference is that with light the propagation speed is too high to measure the time it took for the light to bounce back. Instead, the phase difference of the light, and the fact that the coherence length of the light source is very short, are used to filter out other light that arrives at the detector. This incoherent, i.e. 


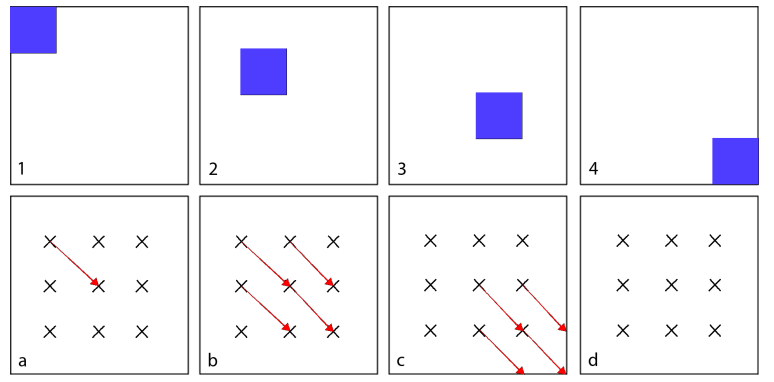

Fig. 2 An illustration of optical flow as a principle. (1-4) The frameby-frame movement of an object in a scene. (a-d) The calculated optical flow vector field by using the expressed 9 points in the image.

wide-spectrum light, allows the obtainment of micrometer-resolution images from samples. Traditionally, OCT machines have worked in the time domain, by scanning over a single column of the tissue by moving a reference mirror. This approach with its moving parts is relatively slow, however, and recently frequency domain OCT (FD-OCT) devices have been manufactured, which allow for real-time capturing of volumetric images with voxel counts in the tens of millions.

\subsection{Optical flow}

Optical flow, in a general sense, is the apparent movement of objects in a scene. In image processing, it is the movement of pixels between two sequential images. An illustration of this is presented in Fig. 2. In this sense, it is often used to track some features over time or space. The general optical flow algorithm is based on two constraints: the brightness constancy constraint [19]:

$E(x, y, t)=E(x+\delta x, y+\delta y, t+\delta t)$,

which essentially confirms that between two sequential samples of a scene (images), the brightness of a certain feature stays unchanged. Another form for the equation is as follows:

$\nabla E \times \mathbf{v}+E_{t}=0$,

where $\nabla E$ is the spatial intensity gradient, and $\mathbf{v}$ the image velocity. This equation has two unknowns and thus cannot be solved as is. Different approaches for calculating optical flow thus introduce additional constraints to the equation. The method used in our approach is known as the Lucas-Kanade method [20], which calculates optical flow locally in a window, overdetermining the above equation, and using the least squares principle to calculate the best optical flow vector. Because it calculates the movement in a local neighbourhood, it is less sensitive to noise than some other optical flow calculation methods. In particular we use an iterative version of the method that utilizes image pyramids and affine transformations for more robust tracking [21]. It was selected over feature- and pure pixel based methods because of the relative robustness to noise (calculates movement in local patches) and large scale movements.

\section{Method}

Five months old laboratory mice were used for cochlea harvesting. In total, two mice had their cochlea harvested, one of which was morphologically normal $\left(\operatorname{Slc} 26 a 4^{(+-)}\right)$, and the remaining presenting endolymphatic hydrops $\left(S l c 26 a 4^{(-/-)}\right)$. Cochlea from both ears were used. After harvesting the cochlea, they were en-

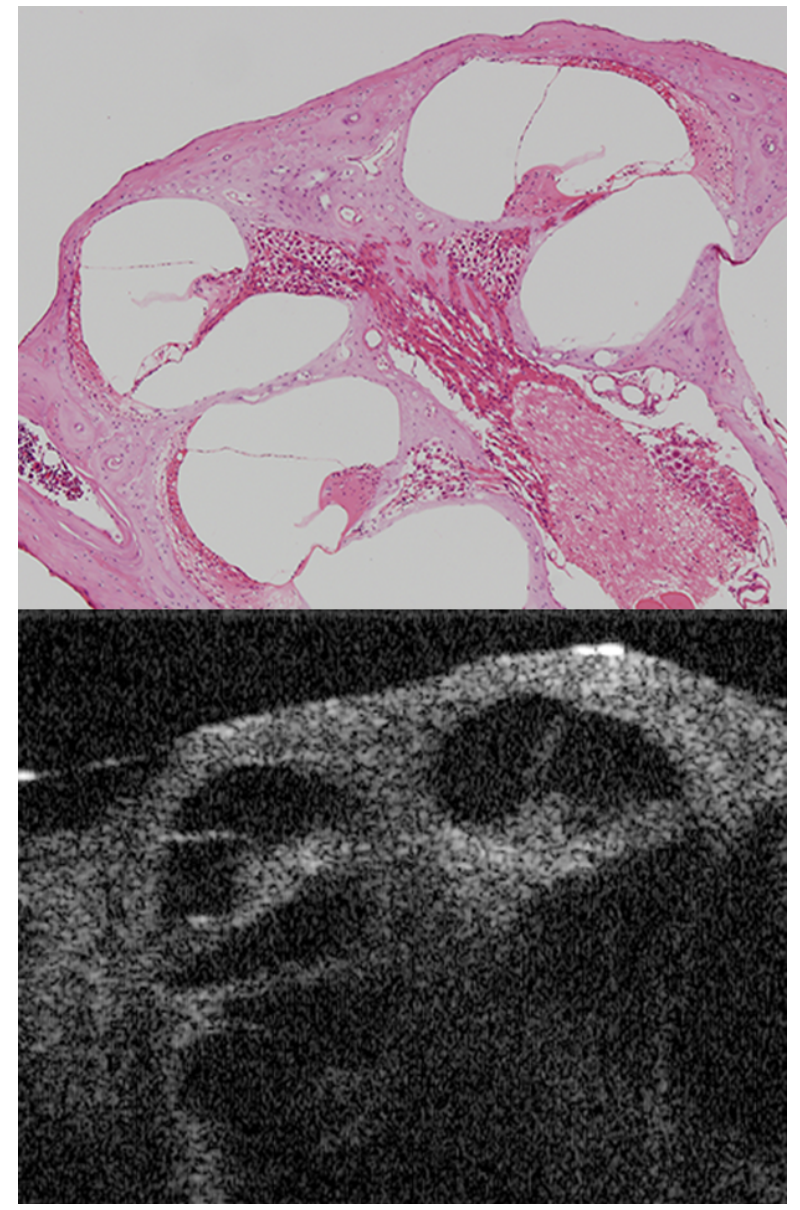

Fig. 3 Upper: Cochlear structures visible in a histological frame. Lower: an OCT slice of the cochlea, from roughly the same area as the above histological image.

cased in a $4 \%$ paraformaldehyde solution overnight, after which they were immersed in $10 \%$ ethylenediaminetetraacetic acid (EDTA) for 7 days for decalcification. After that they were imaged using the OCT machine. Following, they were encased in paraffin solution for a week and histological slices were obtained. 7-10 slices per cochlea were obtained to ensure that the measuring would be a success. The slices were $4 \mu \mathrm{m}$ thick with $20 \mu \mathrm{m}$ spacing. Histological slices were stained using hematoxylin and eosin, and imaged using a microscope (Olympus BX50). From the images obtained, the areas of the particular compartments of the cochlea were calculated using ImageJ software [22] by hand-drawing borders around the three structures, and having the software calculate the areas. The experiment was approved by the animal experiment ethics committee at Kyoto University prior to conducting the experiments (Approval number: MedKyo15155). An example that shows both the OCT image as well as the dyed histological slice from roughly the same area is shown in Fig. 3 .

From the OCT volumetric image series, the angle facing the scala media between the Reissner's membrane and the spiral ligament was calculated using custom software. To obtain a more reliable result for the angle, as a preprocessing step the algorithm applies a bilateral filter to remove noise in the image. Bilateral filter was selected because it is good at reducing noise while maintaining the edges present in the image. Then, the software 


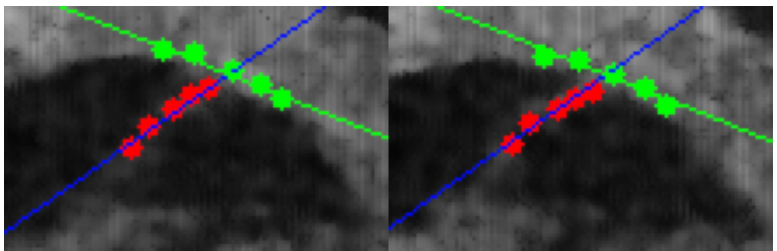

Fig. 4 Example of the apparent movement of the structures between two frames. Due to the oblique angle of the Reissner's membrane in comparison to the axes, it exhibits apparent movement when browsing from frame to frame. The optical flow algorithm keeps track of the position of the membrane.

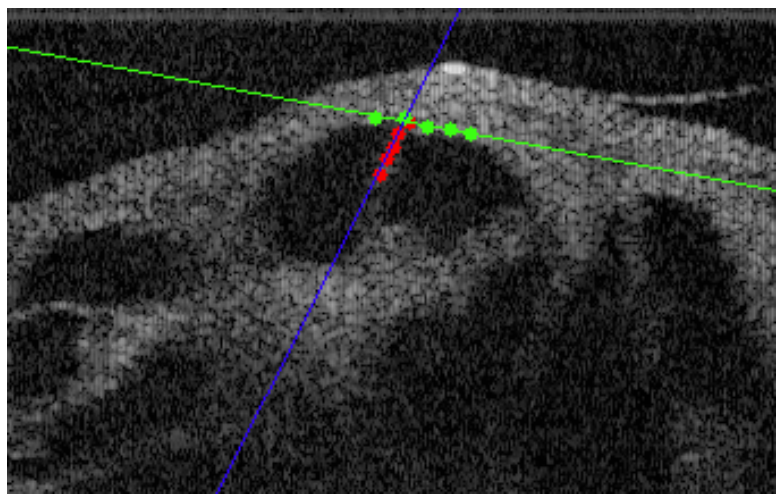

Fig. 5 An example of an OCT image with the user inputted points and the planes the software calculates

prompts the user to draw points on a slice corresponding to the two different structures. From these points, using an optical flow algorithm, the software calculates the movement of the points over several frames to both directions along the Z-axis (refer to Fig. 6). An example of the movement of the points and the tracking by optical flow is illustrated in Fig. 4. This mitigates the effect a single slice has on the angle. As the angle can be hard to see, two rules for the point selection were made: 1) the connection between the Reissner's membrane and the spiral ligament has to be clearly visible, and 2) since the exact point of attachment can be hard to discern, the point of steepest angle around the area is used as the point between the two planes. The slice as close as possible to the center of the cochlea was used, to ensure that all of the structures would be visible, and that the angles would be as close to right as possible. Two planes are then fitted using all of the points, and the angle between the planes is used as the measurement of endolymphatic hydrops. An illustration of the user inputted points as well as the calculated lines can be seen in Fig. 5. From the slices, the ratio between the area of the scala media and the total area of the three vestibules was calculated. Using these two measures, the angle and the ratio were compared to find out if there exists a correlation between the two variables. Also, using the calculated ratio, the degree of hydrops was assigned by using the scale mentioned in section 2.

To summarise, the workflow is as follows:

1. Load the data.

2. Navigate to the center slice of the data.

3. Assuming the structures are clearly visible, mark the points on

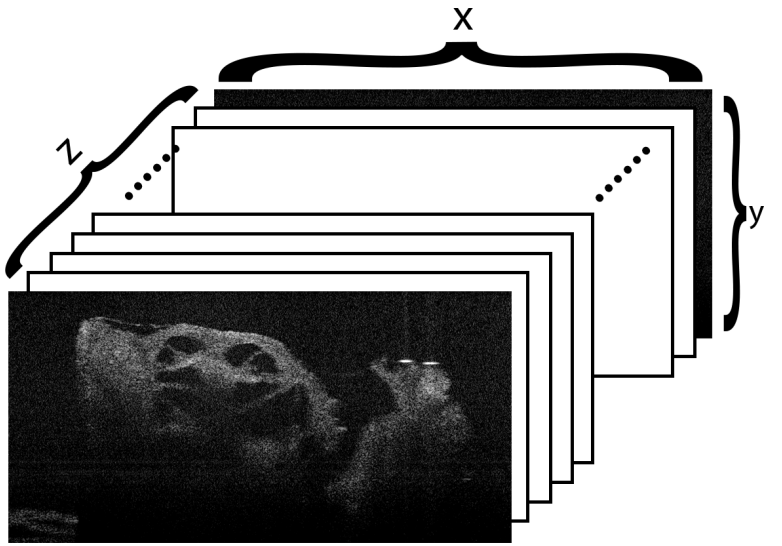

Fig. 6 The dimensions of the captured data. $\mathrm{X}$ is the amount of axial scans, which in our study was 1024 over $6 \mathrm{~mm}$ physical length. $\mathrm{Y}$ is the depth of the scan, and $\mathrm{Z}$ the amount of slices scanned. Both were set as 256 over $1.5 \mathrm{~mm}$.

the two different structures.

4. Calculate the positions on the $n$ adjacent slices.

5. Fit two planes on these points using a least squares plane fitting algorithm.

The size $\&$ resolution of the OCT images obtained are illustrated in Fig. 6. The machine used was a Thorlabs OCS1300SS OCT system, with a central wavelength of $1300 \mathrm{~nm}$, a spectral bandwidth of $100 \mathrm{~nm}$, and an average output power of $10 \mathrm{~mW}$. The axial scan rate of the machine is $16 \mathrm{kHz}$. Thus it can obtain the measured 1024 column by 256 slide volumes in about 16 seconds.

\section{Results}

The results of the measurements are shown in table 1, and further visualized in Fig. 7. The data is comprised of three sets of measurements made by different researchers, as well as the calculated averages and standard deviations. Two of the researchers mentioned were qualified medical professionals, and one was familiar with the proposed method. The grades are assigned according to the method explained in section 2. As can be seen, there is a clear difference between the ratios of the cochlear ducts, as well as the angles, between the healthy and the diseased cochlea. Furthermore, two clear groups can be seen in the scatter plot, indicating that there is indeed correlation between the angle and the ratio.

Using the Kruskal-Wallis test to check if the angles between the normal and the hydrops groups differs gives $\chi^{2}=8.3077$ with $p=0.004$, indicating that the mean ranks between the two groups are different. This suggests that there is a significant difference with the angle between the cochlea with and without hydrops.

Calculating the Pearson correlation coefficient using the values provided gives $R=0.977$ with $p=0.5 \times 10^{-7}$, indicating that there exists a statistically significant correlation between the angle of the Reissner's membrane, and the degree of endolymphatic hydrops as measured by the ratio of the different scalae. However, with the low amount of samples caution should be exercised when interpreting the numbers.

\section{Discussion}

This research shows that the angle between the Reissner's mem- 
Table 1 Results of the measurements. NO = morphologically normal type, $\mathrm{HY}=$ presenting hydrops.

\begin{tabular}{llll}
\hline \hline & ratio [\%] & $\begin{array}{l}\text { angle } \\
{[\mathrm{deg}]}\end{array}$ & $\begin{array}{l}\text { assigned } \\
\text { grade }\end{array}$ \\
\hline NO left & 0.258 & 109.239 & none \\
NO right & 0.270 & 106.195 & $\begin{array}{l}\text { none } \\
\text { severe }\end{array}$ \\
HY left & 0.953 & 150.214 & severe \\
HY right & 0.932 & 148.891 & none \\
\hline NO left & 0.242 & 116.913 & none \\
NO right & 0.267 & 107.191 & severe \\
HY left & 0.946 & 152.723 & severe \\
HY right & 0.923 & 146.321 & none \\
\hline NO left & 0.233 & 108.279 & none \\
NO right & 0.253 & 99.675 & severe \\
HY left & 0.952 & 150.239 & severe \\
HY right & 0.938 & 142.022 & \\
\hline NO mean & 0.254 & \pm 107.915 & \pm \\
\& st. dev. & 0.014 & 5.554 & \\
HY mean & 0.941 & \pm 148.402 & \pm \\
\& st. dev. & 0.012 & 3.758 & \\
\hline & &
\end{tabular}

brane and the outer cochlear wall can be used as a metric for measuring the existence of endolymphatic hydrops using OCT imaging. Traditionally, measuring it in vivo has been difficult. Measuring the degree of endolymphatic hydrops with MRI needs to be carried out with the aid of some shadowing agent, which carries the risk of nephrogenic systemic fibrosis [23] and relies heavily on the clinical expertise of the operating physician to determine the locations and sizes of the various structures inside cochlea. With OCT the structures are also more clear. However, completely imaging the cochlear shape with OCT is difficult because of the rapid drop off in intensity as deeper structures are imaged. Combining OCT with our technique, these measurements are made possible by replacing the traditionally used metric of the ratio with a metric more useful concerning the limitations of OCT. Together with previous research done on in vivo OCT imaging of the cochlea, this suggests the viability of in vivo measurement of endolymphatic hydrops. The fact that OCT does not penetrate hard tissue such as bone very well implies that completely non-invasive visualization cannot be done, and that surgical procedures are needed to gain access to the cochlea.

Despite the correlation present in the results, more data should be collected for more robust testing. Due to the relative simplicity in obtaining mice with and without severe endolymphatic hydrops, the two groups were fairly well separated, as can be seen from Fig. 7. Indeed, using the scale mentioned in section 2 , the measurements would be classified either as none or as severe, depending if they were done on mice with the gene intact, or removed, respectively. Thus, imaging various degrees of hydrops would be fruitful for seeing in what way the data is distributed between these two extremes.

From the data obtained, and as can be seen from Fig. 3, it can be difficult to discern exactly where the Reissner's membrane actually connects to the spiral ligament. In our study, this was distribution of ratio/angle

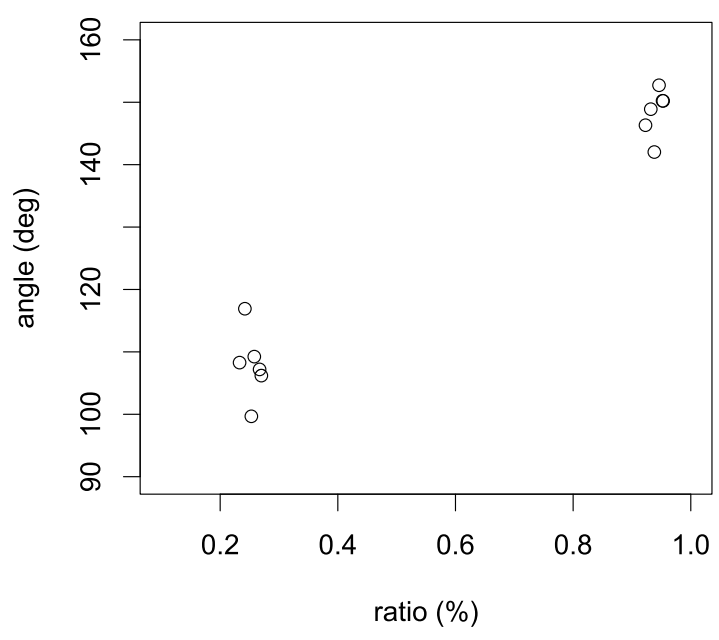

Fig. 7 Plotting the obtained angle \& ratio values in a scatter plot. Two distinct groups can be seen, indicating correlation between the two different measures.

taken into account by starting the two different planes from the sharpest point in the inner curve, but cannot completely be alleviated as the problem raises from the limited resolution \& noise floor of the images. Also, due to the curvature of the Reissner's membrane, the angle depends somewhat on how long of a stretch of the membrane one uses as an input to the algorithm. This is because the algorithm fits a plane to the points. This could be alleviated by trying to fit a parabolic surface instead.

Atrophy of the lateral wall also affects the angle measured, thus modifying the ratio between the angle and the area. Properly determining the effect of lateral wall atrophy on the ratio will require more measurements. In microscope images of the cochlea, the surface of the Reissner's membrane is often not completely smooth, but exhibits irregularities and is sometimes completely separated from one side. It is likely that this is caused by the histological slicing, rather than being actually present in the cochlea before separation. The method presented using OCT and angle calculation is less disruptive to the cochlea, thus in effect giving a higher chance for the membrane to be of normal condition. However, the case where the membrane is disrupted but continuous is a limitation of our method, as the angle is difficult to calculate but area calculation should still be achievable.

More robust handling of noise could also be attempted, as optical flow is considerably sensitive to it. Either a noise model that takes better in to account the multiplicative nature of the noise in OCT images could be developed, such as the one decribed in [24], or a method for tracking the positions that is less sensitive than optical flow to noise could be implemented. The tradeoff for these potentially better performing algorithms likely is speed however. The relatively substantial amount of data that OCT produces makes it costly to do any kind of complicated processing.

Optical flow as a method for object tracking has been used widely and is regarded as a reliable method. Extensive evaluations on the accuracy of different optical flow algorithms (including the one we used) can be found in [25]. Validation of the accuracy of the optical flow method as pertains to the angle 
measurements was considered out of scope for mainly two reasons: 1) the accuracy was deemed sufficient by the doctors that used the application, and 2) accurately measuring the accuracy is in itself a problem with no clear solution, as calculating the movement by hand is very time consuming and will not necessarily produce the right answer.

Future work could also include automatically detecting the position of the Reissner's membrane. This would allow for completely automatic measurement of endolymphatic hydrops from OCT images.

\section{Conclusion}

In this paper, we presented a method for indirectly measuring the degree of endolymphatic hydrops by calculating the angle between the Reissner's membrane and the spiral ligament inside the cochlea as imaged by optical coherence tomography. Preliminary results indicate that there exists a correlation between the angle and the degree of the hydrops.

\section{Acknowledgements}

The authors thank Konrad Noben Trauth, Andrew Griffith, and Eric Green at the National Institute of Health (NIH) for the generous donation of Slc26a4-disrupted mice. Also, the authors would like to thank the Center for Anatomical, Pathological and Forensic Medical Researches, Kyoto University Graduate School of Medicine, for preparing microscope slides.

The authors declare no conflicts of interest relationship with any companies or commercial organizations based on the definition of Japanese Society of Medical and Biological Engineering.

\section{References}

1. Everett LA, Belyantseva IA, Noben-Trauth K, Cantos R, Chen A, Thakkar SI, Hoogstraten-Miller SL, Kachar B, Wu DK, Green ED: Targeted disruption of mouse pds provides insight about the inner-ear defects encountered in pendred syndrome. Human Mol Genet. 10(2), pp. 153-161, 2001.

2. Sajjadi H, Paparella MM: Meniere's disease. Lancet. 372(9636), pp. 406-414, 2008.

3. Nakashima T, Naganawa S, Sugiura M, Teranishi M, Sone M, Hayashi H, Nakata S, Katayama N, Ishida IM: Visualization of endolymphatic hydrops in patients with meniere's disease. Laryngoscope. 117(3), pp. 415-420, 2007.

4. Pyykkö I, Zou J, Poe D, Nakashima T, Naganawa S: Magnetic resonance imaging of the inner ear in meniere's disease. Otolaryngol Clin North Am. 43(5), pp. 1059-1080, 2010.

5. Subhash HM, Davila V, Sun H, Nguyen-Huynh AT, Shi X, Nuttall AL, Wang RK: Volumetric in vivo imaging of microvascular perfusion within the intact cochlea in mice using ultra-high sensitive optical microangiography. Med Imaging, IEEE Trans. 30(2), pp. 224-230, 2011.

6. Tona Y, Sakamoto T, Nakagawa T, Adachi T, Taniguchi M, Torii $\mathrm{H}$, Hamaguchi K, Kitajiri Si, Ito J: In vivo imaging of mouse cochlea by optical coherence tomography. Otol Neurotol. 35(2), pp. e84-e89, 2014.

7. Hallpike CS, Cairns H: Observations on the pathology of meniere's syndrome. Laryngol Otol. 53(10), pp. 625-655, 1938.

8. Yamakawa K: Über die pathologische veränderung bei einem meniere-kranken. J Otorhinolaryngol Soc Jpn. 44, pp. 23102312, 1938.

9. Salt AN, Henson MM, Gewalt SL, Keating AW, DeMott JE, Hen- son O: Detection and quantification of endolymphatic hydrops in the guinea pig cochlea by magnetic resonance microscopy. Hearing Res. 88(1), pp. 79-86, 1995.

10. Nakashima T, Naganawa S, Pyykkö I, Gibson WP, Sone M, Nakata S, Teranishi M: Grading of endolymphatic hydrops using magnetic resonance imaging. Acta Oto-Laryngologica. 129(s560), pp. 5-8, 2009.

11. Kakigi A, Takubo Y, Egami N, Kashio A, Ushio M, Sakamoto T, Yamashita S, Yamasoba T: Evaluation of the internal structure of normal and pathological guinea pig cochleae using optical coherence tomography. Audiol Neurotol. 18(5), pp. 335-343, 2013.

12. Curati W, Graif M, Kingsley D, King T, Scholtz C, Steiner R: Mri in acoustic neuroma: a review of 35 patients. Neuroradiology. 28(3), pp. 208-214, 1986.

13. Hulcelle P, Dooms G, Mathurin P, Cornelis G: Mri assessment of unsuspected dural sinus thrombosis. Neuroradiology. 31(3), pp. 217-221, 1989.

14. Hee MR, Puliafito CA, Wong C, Duker JS, Reichel E, Rutledge B, Schuman JS, Swanson EA, Fujimoto JG: Quantitative assessment of macular edema with optical coherence tomography. Arch Ophthalmol. 113(8), pp. 1019-1029, 1995.

15. Hee MR, Puliafito CA, Wong C, Duker JS, Reichel E, Schuman JS, Swanson EA, Fujimoto JG: Optical coherence tomography of macular holes. Ophthalmology. 102(5), pp. 748-756, 1995.

16. Kafieh R, Rabbani H, Kermani S: A review of algorithms for segmentation of optical coherence tomography from retina. J Med Signals Sensors. 3(1), p. 45, 2013.

17. Gilroy A, MacPherson B, Ross L: Atlas of Anatomy. Thieme Anatomy, Thieme, 2008.

18. National Institute of Health: Meniere's disease. NIH Publication no. 10-3404, 2010.

19. Horn BK, Schunck BG: Determining optical flow. In 1981 Technical symposium east, International Society for Optics and Photonics, 1981, pp. 319-331.

20. Lucas BD, Kanade T, et al.: An iterative image registration technique with an application to stereo vision. In IJCAI, 1981, vol. 81, pp. 674-679.

21. Bouguet JY: Pyramidal implementation of the affine lucas kanade feature tracker description of the algorithm. Intel Corporation. 5, pp. 1-10, 2001.

22. Abràmoff MD, Magalhães $\mathrm{PJ}$, Ram SJ: Image processing with imagej. Biophotonics Int. 11(7), pp. 36-42, 2004.

23. Goenka AH, Das CJ, Sharma R: Nephrogenic systemic fibrosis: a review of the new conundrum. National Medical Journal of India, 2009.

24. Yu Y, Acton ST: Speckle reducing anisotropic diffusion. Image Processing, IEEE Trans. 11(11), pp. 1260-1270, 2002.

25. Baker S, Scharstein D, Lewis J, Roth S, Black MJ, Szeliski R: A database and evaluation methodology for optical flow. Int J Comput Vision. 92(1), pp. 1-31, 2011.

\section{Tuukka Karvonen}

Tuukka Karvonen is a Ph. D. student at Graduate School of Informatics, Kyoto University, Japan. He received his M.Sc. in computer science \& engineering from University of Oulu. His research interests include computer vision, medical imaging and human-computer interaction.

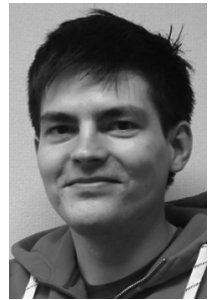




\section{Yuki Uranishi}

Yuki URANISHI is an assistant professor of the Division of Medical Information Technology and Administration Planning at Kyoto University Hospital. He received his $\mathrm{Ph} . \mathrm{D}$. degree in engineering from the Graduate School of Information Science, Nara Institute of Science and Technology, Japan in

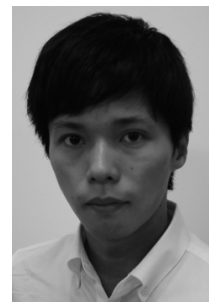
2008. Before joining Kyoto University Hospital, he has been an assistant professor at Nara Institute of Science and Technology, and at Osaka University. His research interests include three-dimensional measurement, augmented reality and human-computer interaction.

\section{Tatsunori SАкамото}

Tatsunori SAKAMOTO is an assistant professor at the Department of Otorhinolaryngology in Kyoto University Hospital. He graduated as Doctor of Medicine in 1995, and Ph.D. in medicine in 2004 from Kyoto University. His previous posts include Kobe City General Hospital and RIKEN institute. His

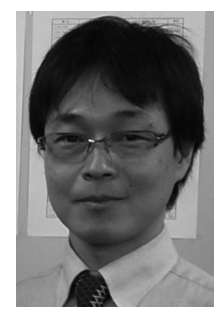
research interests include inner ear regeneration, diagnostic imaging of inner ear and rhinology, as well as inner ear drug delivery systems.

\section{Yosuke TonA}

Yosuke Tona is working as a medical doctor at the Department of Otorhinolaryngology in Kyoto University Hospital. He graduated as Doctor of Medicine in 2005, and is currently working on his Ph.D. with expected graduation in 2016. Before his current post, he worked in Kobe City Medical Center

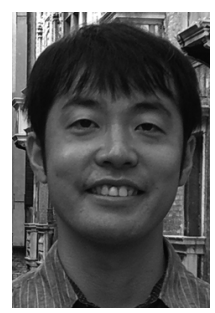
General Hospital, as well as Shizuoka General Hospital. Research interests include inner ear imaging and developmental analysis.

\section{Kazuya Окамото}

Каzuуа Окамото is a lecturer of Division of Medical Information Technology and Administrative Planning of Kyoto University Hospital. He received B.S., M.S. and Ph.D. degrees in informatics from Kyoto University. His current research interests include medical informatics, artificial intelligence in medicine, and rehabilitation engineering.

\section{Hiroshi TAMura}

Hiroshi TAMURA is an associate professor of the Division of Medical Information Technology \& Administration Planning at Kyoto University Hospital. He graduated with Ph.D. from Kyoto University Graduate School of Medicine in 2006. He has previously worked in Kobe City General

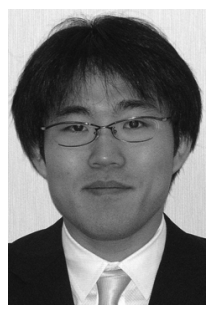
Hospital from 1998-2002 at the department of ophthalmology as junior \& senior resident. Research interests include ophthalmology, age-related macular degeneration, hospital management, epidemiology, and health economics.

\section{Tomohiro KURODA}

Tomohiro KuRODa is the professor of the Division of Medical Information Technology and Administrative Planning of Kyoto University Hospital. He received his $\mathrm{Ph}$. D. in Information Science from Nara Institute of Science and Technology. His research interest includes human interfaces, virtual/

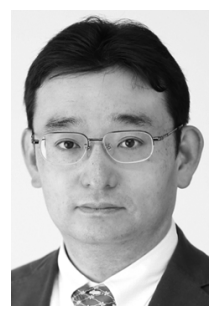
augmented reality, wearable computing, and medical \& assistive informatics. 